\title{
POSITIVITY IN $H$-SYSTEMS AND SUFFICIENT STATISTICS $\left({ }^{1}\right)$
}

\author{
BY \\ T. S. PITCHER( $\left.{ }^{2}\right)$
}

Introduction. In $\S 1$, Theorem 2.1 of $[6]\left(^{3}\right)$ is improved slightly and then is used to show that nonzero, central positivity preserving projections in the $H$-system of a group arise in a simple way from compact normal subgroups. In $\$ 2$ this theorem is applied to a problem in statistics.

1. Let $G$ be a separable, locally compact, unimodular group, $H(G)$ its $H$-system (see [1] and [5] for the definition of an $H$-system), $P$ the nonnegative elements of $H(G)$, and $W(H(G))$ the weakly closed ring of operators generated by left convolution operators in $H(G)$. An element of $W(H(G))$ is central if it commutes with all right convolution operators. If $x$ is an element of $G$ or $f$ is a function on $G$ we write $l(x)$ or $l(f)(r(x)$ or $r(f))$ for the corresponding left (right) translation or convolution operators.

If $K$ is a compact normal subgroup of $G$ and we define $\pi f$ by $\pi f(x)$ $=\int_{K} f(k x) d k$ where $d k$ is normalized Haar measure on $K$ then $\pi$ is a central projection and $\pi P \subset P$. Moreover every $\pi f$ corresponds to a function $i(\pi(f))$ on $G / K$ and $i$ gives a positivity preserving isomorphism between $\pi(H(G)$ ) and the $H$-system of $G / K$. The following theorem shows that every positive central projection arises from such a $K$ in this way.

TheOREм 1.1. Let $\pi$ be a nonzero central projection in $W(H(G))$ with $\pi P \subset P$ and let $K=\{x: x \in G, \pi(x)=\pi\}$. Then $K$ is a compact normal subgroup and $\pi f(x)=\int_{K} f(k x) d k$ almost everywhere for every bounded $f$.

Proof. $K$ is clearly a closed and normal subgroup. We can find a neighborhood $U$ of the identity for which the closure $S$ of $U U^{-1}$ is compact and a function $p$ with $\int_{U}(\pi p)^{2} d x>0$. If a sequence $k_{i}$ from $K$ is defined as follows: $k_{1}=$ identity and $k_{i+1} U$ is disjoint from $\bigcup_{j=1}^{i} k_{j} U$ then we have

$$
\int(\pi p)^{2} d x \geq \sum_{j=1}^{i} \int_{k_{j} U}(\pi p)^{2} d x=i \int_{U}(\pi p)^{2} d x
$$

so the sequence must end for some $N$ and $K \subset \bigcup_{j=1}^{N} k_{j} S$ which is compact.

Hence, if $f$ is bounded and measurable $\rho f(x)=\int_{K} f(k x) d k$ is defined and $\rho$

Received by the editors May 14, 1956.

(1) The research reported in this document was supported jointly by the Army, Navy and Air Force under contract with the Massachusetts Institute of Technology.

(2) Staff Member, M.I.T., Lincoln Laboratory.

(3) Numbers in square brackets refer to the bibliography at the end of the paper. 
can be extended to a central positivity preserving projection of $H(G)$ onto a subset isomorphic to $H(G / K)$ and satisfying $\pi \rho=\rho \pi=\pi . x K \rightarrow l(x)$ maps $G / K$ onto a group of unitaries operating on $\pi H(G)$ and taking $\pi P$ into itself. If we could verify the conditions of Theorem 2.1 of [6] we could infer that $\pi H$ was isomorphic to $H(G / K)$ under the map $i: i(f(x K))=f(x)$, and this would complete the proof since $\rho H$ is also isomorphic to $H(G / K)$ under the same map. However there seems no easy way to verify condition (3) in the definition of an $H P$ system [6, p. 485] so we are forced to prove an alternate version of the theorem.

Let $C=\left\{\sum_{i=1}^{n} p_{i} q_{i} ; p_{i}, q_{i} \in \pi P\right.$ and the products involved are defined $\}$ and $\left[\sum_{i=1}^{n} p_{i} q_{i}\right]$ be the function on $G / K$ defined by:

$$
\left[\sum_{i=1}^{n} p_{i} q_{i}\right](x K)=\sum_{i=1}^{n}\left(p_{i}, l(x) q_{i}\right) .
$$

Lemma 1.1. (1) $\pi H$ can be represented as the $L_{2}$ of some measure space in such a way that $\pi P$ corresponds to the almost everywhere non-negative functions in this $L_{2}$.

(2) $\pi P=(\pi P)^{*}$.

(3) If $f_{i}, h \in C$ and there is an $f \in C$ with $f_{i} \leq f\left(f-f_{i} \in \pi P\right)$ for all $i$ and $\sup \left[f_{i}\right] \geq[h]$ then $\sup f_{i} \geq h$.

(4) There are approximate identities for $\pi H(G)$ in $\pi P$ and $\pi P \cap A$ is dense in $\pi P$, where $A$ is the bounded algebra of $H(G)$ (see [1]).

(5) $C \subset \pi P$.

Proof. If $\pi p$ and $\pi q$ are in $\pi P$ then $(\pi p, \pi q) \geq 0$ since $\pi P \subset P$ and if $(\pi p, \pi x)$ $\geq 0$ for all $\pi p \in \pi P$ then $(p, \pi x) \geq 0$ for all $p \in P$ so $\pi x \in P$ so $\pi x \in \pi P$. Hence by a theorem of Nagy [4] (1) will be verified if we show that whenever $\pi p_{1}, \pi p_{2}$, $\pi q_{1}$, and $\pi q_{2}$ are in $\pi P$ and satisfy $\pi p_{1}+\pi p_{2}=\pi q_{1}+\pi q_{2}$ we can find $w_{i j}(i, j=1,2)$ in $\pi P$ to satisfy $\pi p_{i}=w_{i 1}+w_{i 2}, \pi q_{i}=w_{1 i}+w_{2 i}$. But since $\pi p_{i}, \pi q_{i}$ are in $P$ we can find $u_{i j}$ in $P$ to satisfy these equations and then set $w_{i j}=\pi u_{i j}$.

For $p$ in $P,\left(\pi\left(p^{*}\right) f, g\right)=\left(\pi\left(p^{*} x\right), y\right)=\left(p^{*} x, \pi y\right)=(x, p(\pi y))=(x, \pi(p) y)$ whenever the products involved are defined which shows that $\pi\left(p^{*}\right)=\pi(p)^{*}$ so that $\pi P=\pi\left(P^{*}\right)=(\pi P)^{*}$ verifying (2). (3) and (5) are trivial and the approximate identities in (4) can be obtained by applying $\pi$ to a set of approximate identities for $H(G)$ in $P$.

The proof of Theorem 1.1 is completed by:

THEOREM 1.2. If $H$ is an $H$-system with a subset $P$ and $G$ is a group of unitaries commuting with right multiplications in $H$, and $H, P, G$ satisfy the conditions (1) through (5) imposed on $(\pi H, \pi P, G / K)$ by the previous lemma then $H, P$ is isomorphic to the $H$-system of $G$ under an extension of the map [ ] of $C$ onto functions on $G$.

Proof. We first prove the following lemma. 
Lemma 1.2. If $D$ is a strongly closed subset of $G$ and $U$ is an element not in $D$ then there is an $f$ in $C$ with $[f](U)=1$ and $[f](V) \leq 1-\alpha<1$ for all $V$ in $D$.

Proof. We can take $U$ to be the identity. For some $p_{i}$ 's in $P \cap A$ the strong neighborhood $\left\{V ;\left\|V p_{i}-p_{i}\right\|<\beta, i=1, \cdots, n\right\}$ is disjoint from $D$ and if we take

$$
f=\sum_{i=1}^{n} p_{i} p_{i}^{*} / \sum_{i=1}^{n}\left\|p_{i}\right\|^{2}
$$

we have $[f](I)=1$ and

$$
[f](V)=\sum_{i=1}^{n}\left(p_{i}, V p_{i}\right) / \sum_{i=1}^{n}\left\|p_{i}\right\|^{2} \leq \sum_{i=1}^{n}\left(\left\|p_{i}\right\|^{2}-\frac{1}{2} \beta^{2}\right) / \sum_{i=1}^{n}\left\|p_{i}\right\|^{2} .
$$

From now on the proof follows the proof of Theorem 2.1 in [6], using the definition of $C$ given above instead of that in [6]. We shall only indicate the changes that are necessary. Lemma 2.1 is no longer needed and Lemma 2.2 is trivial. Lemma 2.3 requires the following result.

Lemma 1.3. If $B$ and $B^{\prime}$ are closed, bounded, and disjoint then

$$
\inf \left(d(B), d\left(B^{\prime}\right)\right)=0 .
$$

Proof. For a $V$ in $B^{\prime}$ choose an $f$ in $C$ with $[f](V)=1+\alpha$ and $[f](B) \leq 1-\alpha$, and let $M(V)=\{W ; f(W)>1\}$. For each $U$ in $B$ choose a $g$ in $C$ with $[g](U)$ $=1$ and $[g](M(V)) \leq 1-\delta$. Then if $e_{n}$ is a set of approximate identities in $P$ we have, on $M(V),\left[(f-\inf (f, g)) e_{n}\right] \geq\left[(f-g) e_{n}\right]$ which converges uniformly to $[f]-[g] \geq \delta$ and at $U,\left[(g-\inf (f, g)) e_{n}\right] \geq\left[(g-f) e_{n}\right]$ which converges to $[g]-[f] \geq \alpha$ so that $2 / \delta(f-\inf (f, g)) e_{n}$ covers $M(V)$ and $2 / a(g-\inf (f \cdot g)) e_{n}$ covers a neighborhood $N(U)$ of $U$ for large enough $n$. Hence $(d(M(V))$, $d(N(U))) \leq(4 / \alpha \delta)\left((f-\inf (f, g)) e_{n},(g-\inf (f, g)) e_{n}\right)$ which converges to 0 so that if $N\left(U_{i}\right)$ is a countable subcovering of $B(d(M(V)), d(B)) \leq(d(M(V))$, $\left.\sup d\left(N\left(U_{i}\right)\right)\right)=0$ and then finally by a similar argument $\left(d\left(B^{\prime}\right), d(B)\right)=0$.

Lemma 2.4 and 2.5 go as before but for Lemma 2.6 we need to know that if $S$ is open and bounded then $d(S) \neq 0$. This is an obvious corollary of the following lemma.

Lemma 1.4. If $S$ is open and bounded, $p$ is in $P$ and for every $U$ in $G$ $(p, U d(S))=0$ then $p=0$.

Proof. It will be sufficient to prove $(p, f)=0$ for all $f$ in $C$. We can find a countable covering $S_{n}$ of $G$ with $S_{n} \subset U_{n} S$ for some $U_{n}$ in $G$ and $a_{n}=\sup f(x)$ for $x$ in $S_{n} \leq 2 \inf f(x)$ for $x$ in $S_{n}$. Then $a_{n} d\left(S_{n}\right) \leq 2 f$ so $a=\sup _{n} a_{n} d\left(S_{n}\right) \leq 2 f$. Choose a set $g_{n j}$ from $C$ so that $\left(g_{n j}\right)_{j=1}^{\infty}$ covers $S_{n}$ and $\left\|\sup , g_{n j}-d\left(S_{n}\right)\right\|$ $\leq\left(\beta / 2^{n}\right)$ so that 


$$
\sup _{n, j} a_{n} g_{n j} \leq 2 f+\sum_{n=1}^{\infty} a_{n}\left(\sup _{j} g_{n j}-d\left(S_{n}\right)\right)
$$

where the last sum exists and has norm $\leq \beta$. Hence $f \leq \sup a_{n} g_{n j}$ so

$$
\begin{aligned}
(p, f) & \leq\left(p, \sup a_{n} g_{n j}\right)=\lim _{N \rightarrow \infty}\left(p, \sup _{n \leq N, j=1} a_{\text {to } \infty} a_{n} g_{n j}\right) \\
& \leq \liminf _{N \rightarrow \infty} \sum_{j=1}^{N} a_{n}\left(p, U_{n} d(S)\right)+a_{n}\left(p, \sup _{j} g_{n j}-d\left(S_{n}\right)\right) \\
& \leq \liminf _{N \rightarrow \infty} \sum(\sup f(x))\|p\| \frac{\beta}{2} n \leq \beta(\sup f(x))\|p\|
\end{aligned}
$$

for every $\beta$.

Finally in the proof of Theorem 2.1 only the second paragraph where it is proved that $S T$ is the identity needs revision. For this we show as before that if $E=S T$ then $E=E^{2}$ so that $\left(E^{*}\right)^{2}=E^{*}$ and for any $x$ and bounded $B$ $\left(x-E^{*} x, U d(B)\right)=\left(x-E^{*} x, E(S(c(U B)))\right)=\left(E^{*} x-\left(E^{*}\right)^{2} x, S(c(U B))\right)=0$ so by the above lemma $E^{*}$ is the identity so $E$ is also.

We can restate Theorem 1.1 using ideals instead of projections as follows.

THEOREM 1.3. If $I$ is a two-sided ideal in $H(G)$ which can be represented as the $L_{2}$ of a measure space in such a way that $I \cap P$ corresponds to the almost everywhere non-negative functions then I consists of all the functions which are invariant under $l(k)$ for all $k$ in the compact normal subgroup $K=\{k ; l(k)$ is the identity on $I\}$.

Proof. The projection $\pi$ of $H(G)$ onto $I$ is central since $I$ is two sided and if $p$ is in $P$ and $q$ is in $P \cap I$ then $(\pi p, q)=(p, q) \geq 0$ which shows that $\pi p$ is in $P \cap I$, i.e. that $\pi P \subset P$. Hence Theorem 1.1 applies and completes the proof.

2. Let $S$ be a field( $\left.{ }^{4}\right)$ of subsets of a set $X$ and for any subfield $S_{1}$ of $S$ let $B\left(S_{1}\right)$ be the set of bounded $S_{1}$ measurable functions on $X$.

Definition. If $S_{1}$ is a subfield of $S, f$ is in $B(S)$, and $m$ is a probability measure on $S$ then the conditional expectation of $f$ with respect to $S_{1}$ and $m$ which we write $E\left(f \mid S_{1}, m\right)$ is the element of $B\left(S_{1}\right)$ satisfying for every $g$ in $B\left(S_{1}\right) \int E\left(f \mid S_{1}, m\right) g d m=\int f g d m$. The existence of $E\left(f \mid S_{1}, m\right)$ and its uniqueness to within sets of $m$ measure zero follow from the Radon Nikodym theorem. If $M$ is a set of probability measures and there is an element $h$ of $B\left(S_{1}\right)$ satisfying $h=E\left(f \mid S_{1}, m\right)$ almost everywhere $(m)$ for every $m$ in $M$ we write $h=E\left(f \mid S_{1}, M\right)$. If $E\left(f \mid S_{1}, M\right)$ exists for every $f$ in $B(S)$ we say $S_{1}$ is a suffcient subfield for $M$.

For most statistical purposes it is sufficient to consider only functions measurable $S_{1}$ if $S_{1}$ is sufficient for the set of measures in question (see [2]),

(4) All fields and subfields used are assumed to be Borel fields. 
and hence it is often a considerable simplification of a statistical problem to find nontrivial sufficient subfields and in particular to find the minimal sufficient subfield when one exists.

If $M$ is dominated, i.e. if there is a measure $n$ with respect to which each $m$ in $M$ is absolutely continuous then it can be shown (see [3, Corollary 4]) that $S_{1}$ is sufficient if and only if all the derivatives $d m_{1} / d\left(m_{1}+m_{2}\right)$ for $m_{1}$ and $m_{2}$ in $M$ are measurable $S_{1}$. From this it follows that there is a best sufficient subfield for a dominated $M$, namely the smallest field with respect to which all the above derivatives are measurable, and that any other subfield is measurable if and only if it contains this one up to sets of $m$ measure zero for every $m$ in $M$.

The following facts are easily proved.

Lemma 2.1. The map $f \rightarrow E\left(f \mid S_{1}, m\right)$ is linear and has a unique extension to a projection in $L_{2}(m) . E\left(f \mid S_{1}, m\right)$ can always be so defined that inf $f(x) \leq E\left(f \mid S_{1}, m\right)$ $\leq \sup f(x)$ everywhere and if $S_{1}$ is sufficient for a set $M$ of measures then $E\left(f \mid S_{1}, M\right)$ can be so defined that inf $f(x) \leq E\left(f \mid S_{1}, M\right) \leq \sup f(x)$.

In many statistical problems $X$ is a topological group, $S$ is the field of Borel sets, and $M$ is a dominated set of measures closed under translation. The problem of finding the mean of a Gaussian distribution of known or unknown or partly restricted variance on the real line is of this type. In this section we find an explicit expression for the best sufficient subfield in this case.

Let $G$ be a locally compact, separable, unimodular group, $S_{0}$ the field of Borel sets of $G$ and $V(G)$ the set of bounded Borel measures make into a Banach algebra with convolution for multiplication and norm given by $m=\sup \int f d m$ where the sup is taken over all $f$ in $B\left(S_{0}\right)$ with sup $|f(x)| \leq 1$. We will write $V^{P}$ for the set of non-negative measures in $V(G)$ and $m_{0}$ for the Haar measure of $G$.

Definition. If $x$ is in $G$, we write $l(x) f$ and $r(x) f$ for the functions defined by $(l(x) f)(y)=f(x y),(r(x) f)(y)=f(y x), l(x) m$ and $r(x) m$ for the measures defined by $\int f d(l(x) m)=\int(l(x) f) d m$ and $\int f d(r(x) m)=\int(r(x) f) d m$. If $S$ is contained in $S_{0}$ we write $l(x) S=\left\{x^{-1} A ; A\right.$ is in $\left.S\right\}$ and $r(x) S=\left\{A x^{-1} ; A\right.$ is in $\left.S\right\}$. The following facts are easily proved.

Lemma 2.2. If $f$ is in $B(S)$ then $l(x) f$ is in $B(l(x) S)$ and $r(x) f$ is in $B(r(x) S)$.

$$
E(l(x) f \mid l(x) S, m)=l(x) E(f \mid S, l(x) m)
$$

and

$$
E(r(x) f \mid r(x) S, m)=r(x) E(f \mid S, r(x) m) .
$$

If a subfield $S$ is sufficient for a set $M$ in $V^{P}$ then $l\left(x^{-1}\right) S$ is sufficient for $l(x) M$ and $r\left(x^{-1}\right) S$ is sufficient for $r(x) M$. 
A set $M$ of measures or a subfield $S$ will be called invariant if $l(x) M$ $=r(x) M=M$ or $l(x) S=r(x) S=S$ for every $x$ in $G$.

Lemma 2.3. If $M$ is invariant, $I$ is the smallest two sided ideal containing $M$ and $I^{P}$ is the set of non-negative measures in I then a subfield is sufficient for $M$ if and only if it is sufficient for $I^{P}$.

Proof. Since $M$ is contained in $I^{P}$, sufficiency for $I^{P}$ clearly implies sufficiency for $M$. Suppose $S$ is sufficient for $M$, then for any $m$ in $M, n_{1}$ and $n_{2}$ in $V(G), f$ in $B\left(S_{0}\right)$, and $g$ in $B(S)$ we have, where $\pi f=E(f \mid S, M)$

$$
\begin{aligned}
\int(\pi f) g d\left(n_{1} m n_{2}\right) & =\iiint \pi f(x y z) g(x y z) d n_{1}(x) d m(y) d n_{2}(z) \\
& =\int d n_{1}(x) \int d n_{3}(z) \int(\pi f) g d(l(x) r(z) m) \\
& =\int d n_{1}(x) \int d n_{3}(z) \int f g d(l(x) r(z) m) \\
& =\int f g d\left(n_{1} m n_{2}\right) .
\end{aligned}
$$

It is easy to extend this to finite sums completing the proof.

From now on we deal with a fixed nontrivial invariant dominated subset $M$ of $V^{P}$ and define $I$ and $I^{P}$ as in the above lemma.

LEMMA 2.4. There are elements $m_{n}$ in $I^{P}$ such that for every non-negative, measurable function $f, \int f d m_{0}=\lim \int f d m_{n}$.

Proof. Choose an $m$ in $M$ and an increasing sequence $A_{n}$ of Borel sets of finite Haar measure whose union is $G$. Let $g_{n}$ be the characteristic function of $A_{n}$ and $d_{n}$ the absolutely continuous measure $g_{n}(x) d m_{0}(x)$. Then $m_{n}=m d_{n}$ is in $I^{P}$ and $\int f d m_{n}=\int d m(x) \int_{A_{n}} f(y x) d m_{0}(y)=\int F_{n} d m \rightarrow \int\left(\lim F_{n}\right) d m=\int f d m_{0}$ since $F_{n}$ is increasing.

Since $M$ is dominated it has a best sufficient subfield $S$ and $S$ is invariant by Lemma 2.1. If $f$ is any non-negative function in $L_{2}\left(m_{0}\right)$ and $\pi f=E(f \mid S, M)$ $=E\left(f \mid S, I^{P}\right)$ then $\int \pi f^{2} d m_{0}=\lim \int \pi f^{2} d m_{n} \leq \lim \int f^{2} d m_{n}=\int f^{2} d m_{0}$ so $\pi f$ is also in $L_{2}\left(m_{0}\right)$ and $\pi$ has a unique extension to a projection in $L_{2}\left(m_{0}\right)$. Now $l(x) \pi f$ $=l(x) E(f \mid S, \quad M)=E\left(l(x) f \mid l(x) S, \quad l\left(x^{-1}\right) M\right)=\pi l(x) f$ and similarly $r(x) \pi f$ $=\pi r(x) f$ so $\pi$ is central and since it also preserves non-negativeness we can apply Theorem 1.1 to it.

THEOREM 2.1. Let $M$ be an invariant dominated subset of $V^{P}$ and $K(M)$ $=\{x ; x \in G$ and $l(x) m=m$ for all $m \in M\}$. Then $K(M)$ is a compact normal subgroup of $G$ and $S(M)=\{A ; A \in S$ and $l(k) A=A$ for all $k \in K(M)\}$ is the best sufficient subfield for $M$. Moreover if $f$ is in $B\left(S_{0}\right)$ and we define $\pi f$ by 
$\pi f(x)=\int_{K(M)} f(x k) d m_{1}(k)$ where $m_{1}$ is the Haar measure of $K(M)$ then $\pi f$ $=E(f \mid S(M), m)$ for every $m$ in $M$.

Proof. We only need to identify $K(M)$ with the $K$ of Theorem 1.1, that is with $\{x ; x \in G$ and $l(x) \pi=\pi\}$. If $l(k) \pi=\pi$ and $m$ is in $M$ then, using the fact that $\pi 1=1$ where 1 is the function which is identically 1 on $G$, we have

$$
\begin{aligned}
\int f d(l(k) m) & =\int f 1 d(l(k) m)=\int(\pi f) 1 d(l(k) m)=\int(l(k) \pi f) 1 d m \\
& =\int(\pi f) 1 d m=\int f d m
\end{aligned}
$$

which proves that $K$ is contained in $K(M) . K(M)$ is clearly closed and it is also compact for if we choose $m$ in $M$ and compact $C$ with $m(C)>0$ then a disjoint sequence $\left(k_{1} C, \cdots, k_{N} C\right)$ must be maximal for some $N \leq m(C)^{-1}$ proving that $K$ is contained in the compact set $\bigcup k_{i} C C^{-1}$. Hence the equation defines a $\pi f$ and it is easily verified that $S(M)$ is a sufficient subfield for $I^{P}$ with $\pi f=E\left(f \mid S(M), I^{P}\right)$ so that $S(M)$ contains $S$ and hence $K(M)$ is contained in $K$.

The assumption that $M$ is dominated or at least admits a minimal sufficient statistic cannot be dropped as the following example shows. Let $G$ be the reals mod one and let $M$ be all the translates of the measure $m$ defined by

$$
\int f d m=\frac{1}{2} \int_{0}^{1} f(x) \phi(x) d x+\frac{1}{4} f(0)+\frac{1}{4} f(1 / 2)
$$

for some $\phi$. Then for each $x$ the subfield $S_{x}$ consisting of all sets either containing both $x$ and $x+1 / 2$ or neither is sufficient for $M$ with

$$
E\left(f \mid S_{x}, M\right)(y)= \begin{cases}f(y) & \text { if } y \neq x \text { or } x+1 / 2, \\ 1 / 2(f(x)+f(x+1 / 2)) & \text { if } y=x \text { or } x+1 / 2 .\end{cases}
$$

If $S$ is contained in all $S_{x}$ and we write $d_{x}$ for the characteristic function of the point $x$ then

$$
E(f \mid S, M) \geq E\left(f(x) d_{x} \mid S, M\right)=\frac{1}{2} f(x)\left(d_{x}+d_{x+1}\right.
$$

so

$$
E(f \mid S, M) \geq \frac{1}{2} f(x)+f(x+1 / 2)
$$

and substituting this in $\int E(f \mid S, M) d m=\int f d m$ gives

$$
\int f(x) \phi(x) d x \geqq \int(f(x)+f(x+1 / 2) \phi(x) d x
$$

which is a contradiction since $f$ and $\phi$ are arbitrary. 


\section{BIBLIOGRAPHY}

1. W. Ambrose, The $L_{2}$ system of a unimodular group. I, Trans. Amer. Math. Soc. vol. 65 (1949) pp. 27-48.

2. R. R. Bahadur, Sufficiency and statistical decision functions, Ann. Math. Statist. vol. 25 (1954) pp. 423-462.

3. P. R. Halmos and L. J. Savage, Application of the Radon-Nikodym theorem to the theory of sufficient statistics, Ann. Math. Statist. vol. 20 (1949) pp. 225-241.

4. B. Nagy, On the set of positive functions in $L_{2}$, Ann. of Math. vol. 39 (1938) pp. 1-13.

5. R. Pallu de la Barriere, Algèbras unitaries et espaces de Ambrose, C.R. Acad. Sci. Paris vol. 233 (1951) pp. 997-999.

6. T. Pitcher, Sets of positive functions in H-systems, Trans. Amer. Math. Soc. vol. 77 (1954) pp. 481-489.

Massachusetts Institute of Technology,

Cambridge, Mass. 